\title{
Revisión
}

\section{Infecciones bacterianas en el paciente crítico: revisión de los estudios publicados entre 2006 y 2008}

\author{
PEDRO M. OLAECHEA \\ Unidad de Cuidados Intensivos. Hospital de Galdakao-Usansolo. Galdakao. Vizcaya. España.
}

\begin{abstract}
Se ha realizado una revisión de las publicaciones médicas entre los años 2006 y 2008 sobre infecciones bacterianas que afectan a los pacientes críticos. Se han seleccionado cuatro temas: neumonía adquirida en la comunidad, neumonía asociada a ventilación mecánica, bacteriemia asociada a catéter y nuevos tratamientos antimicrobianos. En neumonía comunitaria se debe seguir las guías de práctica clínica adaptadas localmente, identificar a los pacientes con relacionados con los sistemas sociosanitarios e ingresar a los pacientes en UCI según criterios clínicos, por la ausencia de normativas completamente fiables. Es necesario aprovechar cualquier información microbiológica para el diagnóstico de neumonía asociada a ventilación mecánica. Ante el riesgo de patógenos multirresistentes, hay que iniciar tratamiento combinado y luego pasar a monoterapia ajustada al antibiograma. Se debe implantar medidas de prevención de neumonía asociada a ventilación mecánica y bacteriemias relacionadas con catéter ya establecidas y que hayan resultado eficaces.

El tratamiento empírico de la bacteriemia relacionada con catéter debe ir dirigido contra los patógenos más probables según el lugar de punción. Los nuevos antibióticos recientemente comercializados están dirigidos básicamente hacia bacterias grampositivas resistentes, mientras que para el tratamiento de los bacilos gramnegativos resistentes es necesario combinar el uso de los nuevos antimicrobianos con una nueva valoración
\end{abstract}

Este artículo de revisión es la transcripción de una ponencia presentada con el mismo título en el XLIII Congreso Nacional de la SEMICYUC en Valencia el día 9 de junio.

Correspondencia: Dr. P.M. Olaechea

Unidad de Cuidados Intensivos. Hospital de Galdakao-Usansolo.

B. ${ }^{\circ}$ de Labeaga, s/n. 48960 Galdakao. Vizcaya. España.

Correo electrónico: pedromaria.olaecheaastigarrag@ osakidetza.net

Manuscrito aceptado el 10-11-2008.. de los antibióticos utilizados desde hace años y la posibilidad de elegir distintas formas de administración.

PALABRAS CLAVE: Infecciones bacterianas. Revisión sistemática. Medicina intensiva. Pacientes críticos.

\section{BACTERIAL INFECTIONS IN CRITICALLY ILL PATIENTS: REVIEW OF STUDIES PUBLISHED BETWEEN 2006 AND 2008}

A systematic revision of medical publications between 2006 and 2008 regarding bacterial infections that affect the critical patients was performed. Four subjects were selected: Communityacquired pneumonia, ventilator-associated pneumonia, catheter-related bloodstream infection and new antimicrobial treatments. When dealing with community-acquired pneumonia and due to the absence of completely reliable standards, it is necessary to follow the locally adapted guidelines of clinical practice, to identify patients related to the health-care system and admit patients to the ICU in accordance with the criteria. Regarding the etiological diagnosis of ventilatorassociated pneumonia, any microbiological information available must be used. Due to the risk of multidrug bacteria, combined empiric therapy should be initiated immediately and then monotherapy adjusted to the antibiogram should be established. Already established measures for mechanical ventilation associated pneumonia and catheter-related bacteriemias, which have been effective, should be implemented.

The empirical treatment of catheter-related bacteremia must be directed towards the most probable pathogens according to the puncture site. The most recently sold antibiotics are basically directed towards multidrug gram positive resistant bacteria. However, for the treatment of gram negative resistant bacilli, the use of the new 
antimicrobials must be combined with a new evaluation of the antibiotics that have been used for years and the possibility of choosing different administration forms.

KEY WORDS: Bacterial infections. Systematic review. Intensive medicine. Critically ill patients.

\section{INTRODUCCIÓN}

$\mathrm{Al}$ acercarnos a la literatura médica publicada en los últimos 2 años sobre las infecciones bacterianas en pacientes críticos, podemos observar dos grandes apartados; por un lado, lo referido a la fisiopatología, el diagnóstico, el tratamiento y la prevención de infecciones bacterianas como tales y, por otro, el diagnóstico precoz y el manejo del cuadro de sepsis o shock séptico que estas infecciones producen. En los últimos años se ha publicado gran cantidad de artículos relacionados con el manejo de la sepsis en general, principalmente sobre los marcadores de sepsis y las recomendaciones de la Surviving Sepsis Campaign. En este artículo se presenta una revisión sistemática de la literatura médica referida a las infecciones bacterianas como tales, dejando de lado el amplísimo campo relacionado con sus efectos en la sepsis.

El campo de las infecciones bacterianas en los pacientes críticos, de todos modos, es muy amplio para revisarlo en un solo artículo, por lo que parece necesario elegir ciertos temas que por su actualidad son de mayor interés: neumonía adquirida en la comunidad (NAC), neumonía asociada a ventilación mecánica (NAVM), bacteriemias (fundamentalmente las asociadas a catéter) y nuevos tratamientos antimicrobianos. Sobre estos temas sobrevuela el aspecto más general de la multirresistencia que afecta a todas las infecciones descritas y, sin abordarla directamente como tema aparte, está en el trasfondo de muy buena parte de lo que se está estudiando en estos días.

El objetivo de este artículo es presentar los aspectos más destacados de una revisión sistemática en infecciones bacterianas en medicina intensiva, repasando la literatura médica publicada entre enero de 2006 y junio de 2008.

\section{MÉTODO}

De manera sistemática se ha revisado los aspectos elegidos que aparecen recogidos en las bases de datos OVID-MEDLINE, EMBASE, EBM Reviews, Pascal Biomed y los conjuntos de base de datos IPA (BBDD). Asimismo se ha hecho un repaso de ensayos clínicos en curso publicados en el registro de ensayos clínicos Clinicaltrial.gov.

Para la búsqueda sistemática se ha utilizado las combinaciones de términos: «bacterial infections», "grampositive infections», "gramnegative infections», "community-acquired pneumonia», «ventilator associated pneumonia», "bacteremia», «multidrug resistant bacteria», "catheter-related bacteremia», «antimicrobial-coated catheters», «an- tiseptic-impregnated catheters», «critically ill patients», «intensive care unit», "antibiotic», "antimicrobial treatment», buscando especialmente los ensayos clínicos en fases III y IV, los estudios comparativos, los metaanálisis y las revisiones sistemáticas publicadas entre enero de 2006 y junio de 2008. Solamente se ha tenido en cuenta los artículos a cuyo texto completo el autor ha podido tener acceso, publicados en inglés o castellano y que de una manera $\mathrm{u}$ otra se refieran a infecciones que son frecuentes en pacientes críticos.

Por lo tanto, se asumen como limitaciones la exclusión de otros temas no incluidos en la selección inicial y la intención expresa de hacer una revisión más amplia que profunda, tratando de dar una pincelada sobre aspectos generales de cada cuestión y procurando dar un acercamiento al conjunto de los lectores de Medicina Intensiva más que a los expertos en enfermedades infecciosas. A fin de facilitar la lectura del artículo, de cada tema se ha extraído una serie de mensajes o consejos que tienen la subjetividad del autor y así deben ser interpretados.

\section{NEUMONÍA ADQUIRIDA EN LA COMUNIDAD}

A principios de 2007, la American Thoracic Society (ATS) y la Infectious Diseases Society of America (IDSA) publicaron la actualización de las recomendaciones de diagnóstico y tratamiento de la NAC, que suponen la puesta al día de las guías publicadas 6 y 4 años antes, respectivamente, de forma independiente por ambas sociedades ${ }^{1}$. En esas recomendaciones se hace mención especial a los criterios de ingreso en la unidad de cuidados intensivos (UCI), los métodos diagnósticos y el tratamiento de los pacientes con NAC que pueden requerir ingreso en UCI.

De los temas que se han abordado en los últimos años en la literatura médica sobre NAC, destaca el seguimiento de las guías de práctica clínica, los criterios de ingreso en UCI, las características de los pacientes con NAC pero que tienen relación con sistemas sociosanitarios y el tratamiento antibiótico combinado.

También son de actualidad otros temas como la duración del tratamiento guiado por marcadores de respuesta inflamatoria sistémica, la influencia de la precocidad del tratamiento en la evolución clínica o la aparición de microorganismos resistentes como etiología de la NAC. Aunque estos aspectos son sin duda interesantes, no se los aborda en esta revisión sistemática.

El seguimiento de las guías de práctica clínica de NAC ya ha sido estudiado, desde hace años, en pacientes que ingresan en salas de hospitalización. Además, en el estudio multicéntrico de pacientes con NAC ingresados en UCI, realizado en 33 UCI de nuestro país (estudio CAPUCI), con más de 500 pacientes, se hizo referencia a un incremento de la mortalidad de los pacientes en los que no se seguían, en cuanto al tratamiento, las guías de la IDSA ${ }^{2}$. Del mis- 
mo estudio, en una publicación posterior ${ }^{3}$, se constata que el seguimiento de estas guías se acompaña también de un menor tiempo de ventilación mecánica, en 3 días, con respecto a los pacientes en los que se iniciaba un tratamiento no recomendado.

Otros estudios han puesto énfasis en el seguimiento de las guías de práctica clínica en el tratamiento de pacientes con NAC incluyendo aquellos que requieren ingreso en UCI, demostrándose una menor mortalidad $^{4-6}$ utilizando guías internacionales ${ }^{4}$, nacionales ${ }^{5}$ o realizadas en el propio hospital ${ }^{6}$.

\section{Criterios de ingreso en UCI de pacientes con NAC}

Otro aspecto de debate en los últimos años lo constituyen los criterios empleados para el ingreso de pacientes en la UCI. Hace tiempo que se han descrito los factores de riesgo asociados con una mayor gravedad de la NAC, reflejados en varias escalas de gravedad aparecidas a lo largo de los años como el Pneumonia Severity Index (PSI), el CURB o el CURB-65, aunque los criterios de recomendación de ingreso en UCI son más dudosos. La propia ATS ha adoptado unos criterios denominados criterios de ATS modificados que miden la gravedad de la enfermedad, y recomienda el ingreso en UCI de los pacientes que requieran ventilación mecánica o fármacos vasoactivos por shock séptico (recomendación fuerte, nivel de evidencia II) o tres de los criterios menores ampliados en la actual revisión ${ }^{1}$. Sin embargo, estos criterios no han sido validados y ninguna de las normas publicadas hasta la actualidad permite distinguir inequívocamente a los pacientes que deben ser ingresados en UCI de los que no.

Para aportar más información, Valencia et $\mathrm{al}^{7}$ describen a la población de pacientes con PSI clase V de su hospital y tratan de identificar qué escala es la más útil para predecir el ingreso en UCI. Esos son pacientes con la mayor gravedad, por lo que sería lógico pensar que deben ser admitidos en UCI en una mayor proporción. Sin embargo, solamente el $20 \%$ de sus 457 pacientes de la clase V del PSI ingresaron en UCI, y son precisamente pacientes más jóvenes y con menos comorbilidades. Los criterios de la ATS modificados ${ }^{1}$ se aproximan más a la predicción de la mortalidad y la necesidad de ingreso en UCI, mientras que el PSI predice bien la mortalidad, pero no es útil para indicar el lugar de atención del paciente.

En los últimos 2 años se han publicado dos estudios en que se compara a los pacientes ingresados en las salas de hospitalización con los ingresados en $\mathrm{UCI}^{8,9}$. En ambos estudios se comprueba que algunos pacientes con poca gravedad medida por el PSI deben ser ingresados en UCI y tienen una mortalidad considerable, y lo contrario, es decir, que pacientes con grados elevados en la escala de PSI (clases IV y V) son ingresados en planta e igualmente tienen una probabilidad de fallecer considerable.

La razón de la discrepancia entre las escalas de gravedad y la necesidad de ingreso en UCI probablemente se deba a que en las escalas no se identifica a los pacientes que previamente tenían relación con los sistemas sociosanitarios ${ }^{10}$. Se define a estos pacientes como los que reciben tratamientos intravenosos en su domicilio, están en hemodiálisis o quimioterapia, han sido admitidos en el hospital por más de 2 días en los últimos 90 antes de la NAC o residen en centros de asistencia sociosanitaria ${ }^{11,12}$. Estos pacientes, según el estudio de Carratala et al $^{13}$, son más ancianos, tienen más comorbilidades, son clasificados como de mayor riesgo y tienen además una mayor proporción de neumococos resistentes a penicilina y macrólidos, por lo que con mayor frecuencia reciben tratamientos inapropiados y, en consecuencia, tienen mayor mortalidad.

\section{Tratamiento antibiótico combinado}

En las guías internacionales ${ }^{1}$ y nacionales ${ }^{14}$, se recomienda que los pacientes con NAC que precisen ingreso en UCI sean tratados con terapia antibiótica combinada para cubrir tanto bacterias atípicas como específicamente Streptococcus pneumoniae o Pseudomonas aeruginosa en pacientes que tengan factores de riesgo de infecciones por estos patógenos. Desde el principio de esta década se han publicado varios trabajos retrospectivos en los que se indica que el tratamiento de la neumonía neumocócica bacteriémica debe realizarse con dos antibióticos, al menos durante unos días.

Sin embargo, es posible que no todos los pacientes que ingresen en UCI por una NAC precisen tratamiento combinado. Así, Rodríguez et $\mathrm{al}^{15}$, con los datos del estudio CAPUCI, encuentran que los pacientes que al ingreso en UCI presentan shock séptico y son tratados con monoterapia tienen una mortalidad superior a la de los pacientes en las mismas condiciones clínicas sometidos a tratamiento combinado ( $h a-$ zard ratio $[\mathrm{HR}]=1,69$; intervalo de confianza [IC] del $95 \%, 1,09-2,6 ; p=0,01)$. Sin embargo, no hay diferencias en la mortalidad en los pacientes con monoterapia o tratamiento combinado en ausencia de shock. Por otro lado, en el estudio de Calzada et $\mathrm{al}^{5}$ el tratamiento de estos pacientes mediante monoterapia con betalactámicos se asocia a un mayor porcentaje de reingresos hospitalarios.

En pacientes no ingresados en UCI, la cobertura de bacterias atípicas no parece que se acompañe de un beneficio en la respuesta clínica ni en la supervivencia, a juzgar por la reciente revisión de la Cochrane Colaboration ${ }^{16}$. Sin embargo, en esta revisión no se ha incluido ningún estudio español ni de pacientes exclusivamente ingresados en UCI, por lo que las conclusiones no pueden extrapolarse a los pacientes de nuestro entorno que requieren ingreso en nuestras unidades.

Mortensen et $\mathrm{al}^{17}$, en un ensayo que refería un incremento de la mortalidad (odds ratio $[\mathrm{OR}]=2,71$; IC del 95\%, 1,2-6,1) entre los pacientes que habían recibido quinolonas más betalactámicos en comparación con los tratados con otras combinaciones de antibióticos, plantearon la cuestión de si los macrólidos tienen un efecto distinto de su efecto antibiótico (in- 
munomodulador o antiinflamatorio) que haría que resultaran más beneficiosos para los pacientes con NAC y sepsis. Dos análisis retrospectivos publicados recientemente irían a favor del empleo de macróli$\operatorname{dos}^{18,19}$. En el primero, Lodise et al refieren que los pacientes con PSI clase V que fueron tratados con macrólidos tuvieron una mortalidad a los 30 días menor que la de los pacientes tratados con quinolonas (el $18,4$ frente al 36\%; $p=0,05)$, mientras que en el segundo, Metersky et al señalan el efecto protector de los macrólidos contra la mortalidad cuando se emplean en el tratamiento de la NAC grave $(\mathrm{OR}=0,68$; IC del 95\%, 0,43-0,87; $p=0,004)$. Un estudio multicéntrico español ${ }^{20}$ no encontró influencia del distinto tratamiento en la evolución de pacientes con neumonía neumocócica, excepto en aquellos con PSI más altos, en los que otras combinaciones de antibióticos (en desacuerdo con las recomendaciones publicadas) se asociarían a mayor mortalidad.

Se ha estudiado el posible beneficio de la claritromicina en pacientes con NAVM y sepsis causada por bacilos gramnegativos resistentes, contra los que la claritromicina no tiene efecto antibiótico ${ }^{21}$. Los pacientes tratados con el macrólido (1 g/día durante 3 días) tuvieron una resolución de la neumonía más rápida que los que recibieron placebo, y por ello el tiempo de ventilación mecánica fue menor. No hubo diferencias en la mortalidad a los 28 días. Parece que la claritomicina pudiera tener un efecto de mejoría de la función pulmonar inhibiendo el reclutamiento de neutrófilos y la producción de interleucina 8 , además de inhibir la producción de quorum sensing de $P$. aeruginosa que coloniza las vías respiratorias. No se sabe aún si este efecto es igual para todos los macrólidos y la influencia que puede tener en el tratamiento de los pacientes sépticos con neumonía u otras infecciones, pero favorecería el empleo de macrólidos en la NAC, por lo que habría que equilibrar este posible efecto con la proporción de resistencias, sobre todo de $S$. pneumoniae.

En la tabla 1 se expresan los mensajes personales extraídos de la bibliografía recientemente publicada sobre NAC.

\section{NEUMONÍA ASOCIADA A VENTILACIÓN MECÁNICA}

Con respecto a la NAVM, parece que actualmente se ha superado la fase de investigación sobre los mecanismos patogénicos y de transmisión de la infección, así como parte de la dudas sobre los métodos diagnósticos, aunque merece la pena hacer una referencia a este apartado, así como tener en consideración los últimos estudios sobre epidemiología básicamente centrada en la multirresistencia, el tratamiento combinado y la prevención de la NAVM.

En un ensayo multicéntrico canadiense de 740 casos de $\mathrm{NAVM}^{22}$, se comparó la evolución y el uso de antibióticos en los casos diagnosticados mediante lavado broncoalveolar (LB) y los diagnosticados mediante aspirado traqueal (AT) no cuantitativo. No hubo diferencias en ambas variables principales del

\section{TABLA 1. Mensajes obtenidos de la revisión sistemática. Neumonía adquirida en la comunidad (NAC)}

Guías de práctica clínica: Seguir escrupulosamente las guías de práctica clínica de NAC adaptadas al entorno

Criterios de ingreso en UCI: No hay ninguna norma fija en los criterios de ingreso de pacientes con NAC en UCI

Pacientes con relación con sistemas sociosanitarios: Es necesario buscar activamente a los pacientes que han tenido contacto con el sistema sanitario. Requieren tratamientos y atenciones diferentes

Tratamiento combinado: Probablemente de inicio en todos los pacientes con NAC que ingresen en UCI. Seguro en todos los pacientes en shock

¿Quinolonas o macrólidos?: Se debe conseguir un balance entre la resistencia a macrólidos y sus posibles efectos inmunomoduladores

estudio (mortalidad del $18 \%$ y cumplimiento del objetivo terapéutico del $74 \%$ en ambos grupos). La principal limitación de este estudio, en lo que pudiera ser extrapolable a nuestro país, es que el porcentaje de patógenos resistentes es muy bajo. En el extremo opuesto, en cuanto a multirresistencia, se ha publicado un ensayo realizado en $\mathrm{Grecia}^{23}$ con una alta proporción de $P$. aeruginosa y $S$. aureus resistente a meticilina (SARM) en el que se comparó la estrategia de desescalamiento antibiótico seguida tras el diagnóstico de la NAVM mediante AT cuantitativo o LB. En los pacientes diagnosticados mediante AT se realizó desescalamiento en el $21 \%$, mientras que en los casos que se llegó al diagnóstico mediante LB el desescalamiento se realizó en el $66 \%$; una vez realizado el ajuste del tratamiento, la evolución fue similar en ambos grupos. Por lo tanto, ese trabajo enseña que los médicos se sienten con más confianza sobre la etiología de la NAVM realizada mediante LB que con AT cuantitativo, lo que lleva a realizar ajustes del tratamiento antibiótico con más frecuencia.

En el último metaanálisis publicado sobre la adecuación del tratamiento antibiótico en NAVM, se vuelve a confirmar que el tratamiento empírico inapropiado se asocia con un incremento de la mortali$\operatorname{dad}^{24}$. En los últimos 2 años se ha estudiado profusamente las causas de que se inicie un tratamiento empírico no adecuado y, por lo tanto, se incremente la mortalidad. Entre estas causas, la principal es la presencia de patógenos multirresistentes ${ }^{25}$. Por lo tanto, los esfuerzos de los investigadores se dirigen a identificar a los pacientes que están en riesgo de sufrir infecciones por estos patógenos multirresistentes para plantear unas pautas terapéuticas con mayor cobertura. En nuestro medio, de los patógenos que causan NAVM, son tres los microorganismos que generan problemas de resistencia, como es el caso de SARM, o de multirresistencia, como $P$. aeruginosa y Acinetobacter baumanii. Medina et $\mathrm{al}^{26}$ han estudiado los factores de riesgo de aislamiento de A. baumanii en una cohorte de 49 pacientes con NAVM por este patógeno, y destacan que el principal factor de riesgo es la utilización previa de antibióticos como 
ceftriaxona o ciprofloxacino. Este aspecto, la relación entre el consumo de ciertos antibióticos y la aparición de patógenos multirresistentes, se está estudiando actualmente con gran intensidad.

Se han publicado en los últimos 2 años numerosos trabajos que estudian los factores de riesgo de aislamiento de $P$. aeruginosa multirresistente ${ }^{27-33}$ y en todos ellos se destaca dos factores: el tiempo de estancia y el empleo de antibióticos previo al episodio de NAVM. Aunque el primero de estos factores no puede evitarse y se incrementa a medida que aumenta nuestra capacidad para disminuir la mortalidad precoz de los pacientes, el segundo -es decir, la estrategia antibiótica-podría tener una influencia teóricamente modificable, por lo que es conveniente profundizar en su conocimiento. Lodise et $\mathrm{al}^{34}$ han buscado parámetros clínicos de la influencia del empleo de antibióticos en la aparición de $P$. aeruginosa multirresistente (definida por resistencia a cuatro o más familias de antibióticos antipseudomónicos). La proporción de patógenos multirresistentes se incrementa desde el $21 \%$ en pacientes que no habían recibido antibióticos hasta el $100 \%$ en pacientes que habían recibido cinco antibióticos previamente. Al estudiar el tipo de antibiótico antipseudomónico implicado en la aparición de multirresistencia, se descubre que todos los antibióticos tienen influencia, pero que depende del tiempo de utilización y que éste es menor para carbapenémicos y fluoroquinolonas $(3 \mathrm{y}$ 4 días respectivamente) que para cefepima (9 días) o piperacilina-tazobactam (12 días). Esto es particularmente significativo cuando se combina con el otro factor de influencia en la aparición de resistencia, el tiempo de estancia prolongada previa a la NAVM. Aunque estos datos se extrajeron de un estudio en un solo centro, lo que supone una limitación, probablemente sean aplicables a nuestro entorno.

Además de los dos factores mencionados, en el caso de SARM se añade otro factor, bien cuantificado desde hace tiempo (y menos estudiado para otros microorganismos): la presencia simultánea de otros pacientes con infección o colonización por este patógeno que comparten la misma área de hospitalización ${ }^{35}$.

A la hora de plantear un tratamiento empírico de NAVM no puede olvidarse el grado de multirresistencia en el entorno en que se halla la unidad. Del estudio ENVIN-UCI ${ }^{36}$, se desprende que la resistencia a ciprofloxacino de Escherichia coli supera, en el total de nuestras UCI, el 30\%, que la resistencia de Acinetobacter baumanii a imipenem es superior al $55 \%$ y que las resistencias de $P$. aeruginosa a diversos antipseudomónicos como ceftazidima, ciprofloxacino, imipenem o piperacilina-tazobactam oscila entre el 25 y el 30\%. Teniendo en cuenta que el objetivo debe ser administrar un tratamiento empírico apropiado, parece razonable que éste sea combinando.

\section{Tratamiento combinado}

Se ha estudiado en los últimos 2 años si es necesario o no utilizar tratamientos combinados para las infecciones por $P$. aeruginosa, lo que hasta ahora constituía casi un dogma. En un reciente metaanálisis sobre tratamientos combinados en NAVM, Aarts et $\mathrm{al}^{37}$ concluyen que la monoterapia no se acompaña de un mayor porcentaje de fallos terapéuticos. Más específicamente, en el tratamiento de NAVM por P. aeruginosa, Garnacho et a ${ }^{38}$ han publicado los resultados de un estudio retrospectivo multicéntrico en el que se recogen 183 NAVM causadas por este patógeno y estudian la evolución de los pacientes según el tratamiento inicial, ya fuera en monoterapia (67) o tratamiento combinado (116). El porcentaje de tratamientos antimicrobianos apropiados con la terapia combinada fue mayor (el 90,5 frente al 56,7\%; $\mathrm{p}<0,001)$, pero si el tratamiento era apropiado no había diferencias en la evolución tanto en monoterapia como en terapia combinada (mortalidad, el 32,1 y el $30 \%$ respectivamente), lo que indica que, una vez conocida la sensibilidad, completar el tratamiento en monoterapia es seguro. Las mismas conclusiones pueden desprenderse del trabajo multicéntrico canadiense $^{22,39}$ en el que se comparó el empleo de meropenem solo o combinado con ciprofloxacino. En el conjunto de NAVM, se obtenía una mayor proporción de tratamientos adecuados con terapia combinada (el 93,1 frente al 85,1\%; $p=0,01$ ), pero sin diferencias en la mortalidad (riesgo relativo $[\mathrm{RR}]=1,05$; IC del $95 \%, 0,78-1,42 ; \mathrm{p}=0,74)$, mientras que cuando se seleccionaban los causados por bacilos gramnegativos (sólo 56 de 740) había mayor porcentaje de tratamiento adecuado (el 84,2 frente al 18,8\%; $\mathrm{p}=0,001$ ) y de respuesta microbiológica en la terapia combinada, pero no había diferencias en la evolución (mortalidad, el 33,3 frente al 41,2\%). Estas conclusiones se recogen en las recomendaciones publicadas por el Comité de Guías de NAVM y el Grupo Canadiense de Ensayos Clínicos en Cuidados $\mathrm{Críticos}^{40}$, que recomiendan iniciar tratamiento empírico con monoterapia que cubra adecuadamente el espectro de patógenos esperados. No debemos olvidar que la etiología de las NAVM en este estudio es diferente de las que ocurren en nuestro entorno, en el que $P$. aeruginosa es el microorganismo más prevalente en las $\mathrm{NAVM}^{36}$, por lo que el tratamiento empírico debe dirigirse a cubrir ese microorganismo en la mayoría de las ocasiones, y con tratamiento combinado ya que es más probable acertar con la sensibilidad.

\section{Prevención de la NAVM}

Otro aspecto importante en relación con NAVM es la prevención, tema que en el futuro seguramente ocupará buena parte de la literatura médica relacionada con esta infección. Puesto que la mayoría de las NAVM se producen por aspiración del contenido orofaríngeo alrededor del tubo endotraqueal, las intervenciones deben encaminarse a conseguir la descontaminación orofaríngea o modificar la composición de los tubos de intubación para que disminuya la posibilidad de aspiración o se retrase la colonización del dispositivo.

En la descontaminación orofaríngea se emplean dos procedimientos distintos, la descontaminación 
con antisépticos o mediante antibióticos dentro del procedimiento más amplio de descontaminación digestiva selectiva (DDS). Hasta el momento no se han hecho estudios que comparen una técnica con la otra. Un primer metaanálisis de Pineda et $\mathrm{al}^{41}$ no encuentra beneficios en la disminución de la incidencia de NAVM ni en la mortalidad con el empleo de clorhexidina oral con respecto a placebo o antisépticos para la cavidad oral. Sin embargo, otros dos metaanálisis posteriores ${ }^{42,43}$ encuentran reducción de la tasa de NAVM (comparada con no hacer profilaxis), aunque sin reducción en los días de ventilación mecánica ni en la mortalidad. En el metaanálisis de Chan et $\mathrm{al}^{43}$, además se estudia los ensayos clínicos en que se realizó profilaxis con aplicación local de antibióticos, y no encuentran diferencias en la tasa de aparición de NAVM (cuatro ensayos: $R R=0,69$; IC del 95\%, 0,41-1,18), pero sí las hubo con el empleo local de antisépticos (siete ensayos: RR = 0,56; IC del 95\%, 0,39-0,81). Esta tasa también es menor en pacientes sometidos a cirugía cardíaca ${ }^{44}$. En otro estudio también se encuentra beneficio con una mezcla de clorhexidina y colistina ${ }^{45}$, aunque igualmente no se hallan diferencias en la estancia en UCI, el tiempo de ventilación mecánica y la mortalidad, pero según los autores añadir colistina al antiséptico puede favorecer la aparición de resistencias a ese antimicrobiano. Seguramente estas medidas de antisepsia deban acompañarse de una limpieza activa de la cavidad oral $^{46}$.

En el otro gran capítulo de prevención de NAVM como es la DDS, sus defensores continúan propugnando sus ventajas ${ }^{47}$ en disminución tanto de la incidencia de NAVM como de la mortalidad ${ }^{48}$. Sin embargo, a la hora de recomendar su uso, sigue levantando suspicacias por los «inconstantes efectos en la mortalidad» y la posibilidad de aparición de resistencias a los antibióticos empleados ${ }^{49}$. Con respecto a este aspecto, Heininger et $\mathrm{al}^{50}$ refieren su experiencia tras 5 años de uso sistemático de DDS, y no encuentran incremento de la resistencia a los antibióticos empleados, aunque sí un incremento de la frecuencia de aislamientos de Staphylococci coagulasa negativos y Enterococci. Por otro lado, se ha publicado la aparición de un brote de bacilos gramnegativos productores de betalactamasa de espectro extendido, lo que enfatiza la necesidad de mantener una actitud de vigilancia de aparición de multirresistencias en unidades en las que se aplique DDS ${ }^{51}$.

Con respecto a otras medidas de prevención relacionadas con los sistemas de aspiración, no parece que las técnicas de aspiración cerrada se acompañen de una disminución significativa de la tasa de NAVM ${ }^{52-55}$, a juzgar por las cuatro revisiones publicadas recientemente, y no es más que la traducción de la escasa proporción de neumonías exógenas con respecto a la de neumonías endógenas, que reflejan el mecanismo patogénico de aspiración del contenido orofaríngeo.

Otro aspecto del cuidado de la vía aérea que se está modificando se refiere a la estructura y la composición de los tubos endotraqueales. Aparte del efecto de prevención de NAVM mediante los sistemas de aspiración de drenaje subglótico (del que no se han publicado nuevos estudios en los últimos 2 años), se está probando modificaciones en la composición del balón de neumotaponamiento para hacerlo más delgado y que se adapte mejor a las paredes de la traquea, con lo que se evita el paso de secreciones entre uno y la otra, como es el empleo de poliuretano. Lorente et $\mathrm{al}^{56}$ encuentran una reducción significativa de NAVM en pacientes en los que se emplea tubos con balones de poliuretano y aspiración subglótica (el 22,1 frente al 7,1\%; $\mathrm{p}=0,001$ ) con respecto a los tubos convencionales, mientras que Poelaert et $\mathrm{al}^{57}$ describen el mismo efecto en pacientes de cirugía cardíaca en los que se utilizan tubos con el balón de poliuretano (el 23 frente al 42\%; p < 0,03), aunque sin aspiración subglótica.

El siguiente paso con respecto a la prevención y la composición de los tubos será fabricarlos recubiertos de materiales que impidan o retrasen la colonización por los patógenos causantes de $\mathrm{NAVM}^{58}$ y que probablemente veremos en los próximos meses un estudio realizado al respecto.

Todas las medidas de prevención de NAVM se han resumido en dos revisiones de Gastmeier et al ${ }^{59} \mathrm{y}$ Lorente et $\mathrm{al}^{60}$, en las que se constata que las oportunidades de disminuir la incidencia de NAVM se basan en medidas simples abordadas desde un punto de vista múltiple, como seguimientos epidemiológicos, educación u otras estrategias de cuidado general de los pacientes. Según la revisión alemana ${ }^{59}$, se podría evitar hasta el $40 \%$ de las infecciones utilizando estas medidas.

En la tabla 2 se recogen algunas recomendaciones personales extraídas de la literatura reciente y referidas a NAVM.

\section{BACTERIEMIA ASOCIADA A CATÉTER}

Como ocurría con la NAVM, en los últimos años se ha dado por bueno los estudios sobre patogenia de la bacteriemia relacionada con catéter (BRC) y en la actualidad están en estudio algunos aspectos de epidemiología, diagnóstico y, sobre todo, prevención. También se está investigando la influencia que pueda tener la formación de biocapas en el desarrollo y la

\section{TABLA 2. Mensajes obtenidos de la revisión sistemática. Neumonía asociada a ventilación mecánica (NAVM)}

Diagnóstico: Aprovéchese la información microbiológica de que se disponga

Epidemiología: Piénsese en los factores de riesgo de aparición de patógenos multirresistentes, sobre todo el tiempo de estancia y el previo empleo de antibióticos

Tratamiento combinado: Iníciese tratamiento combinado ante el riesgo de aparición de patógenos multirresistentes. Ajústese el tratamiento a la monoterapia adecuada

Prevención de NAVM: Medidas sencillas de cuidado de vía aérea superior, programas de educación. Valórese la utilización de tubos con aspiración subglótica y neumotaponamiento de poliuretano, así como la descontaminación digestiva selectiva 
permanencia de las infecciones relacionadas con catéteres y sondas, pero esos estudios aún están en el campo de la microbiología y su repercusión en la clínica todavía está algo alejada.

Con respecto a la epidemiología, el trabajo de Lorente et $\mathrm{al}^{61}$ compara la etiología de la bacteriemia relacionada con catéter según la localización de su inserción y demuestra un mayor porcentaje de aislamientos de bacilos gramnegativos (el 38,9 frente al $7,7 \%$; OR = 7,5; IC del 95\%, 2,2-25,5; p < 0,001) y hongos (el 16,7 frente al 1,9\%; OR =10,2; IC del $95 \%, 1,2-88,8 ; \mathrm{p}=0,035)$ cuando el catéter se localiza en la vena femoral con respecto a otras localizaciones.

Aunque durante muchos años se ha estudiado los diferentes métodos para el diagnóstico de las BRC, lo cierto es que la aplicación en clínica de estos métodos no se ha extendido en nuestra práctica clínica y en pacientes en UCI sigue siendo más fácil y segura la sustitución del catéter que darle mantenimiento. Por otro lado, se sigue investigando métodos de diagnóstico microbiológico más rápidos (mediante detección de ADN) que permitirían iniciar un tratamiento precoz en caso de que el resultado fuera positivo, aunque aún está lejos su uso habitual. Bouza et $a^{62}$ estudian la utilidad de las tinciones de naranja de acridina y de Gram de la punta del catéter, métodos que son más rápidos que los cultivos, tienen adecuadas sensibilidad y especificidad para el diagnóstico de la infección por catéter y pueden ayudar en la toma de decisiones de tratar o no a un paciente al que se ha retirado el catéter. Los mismos autores ${ }^{63}$ estudiaron tres métodos diferentes de diagnóstico microbiológico sin retirada del catéter en pacientes ingresados en UCI. Por su facilidad y su bajo coste, los autores recomiendan realizar cultivos semicuantitativos de las superficies combinados con cultivos de sangre periférica.

\section{Prevención de la bacteriemia asociada a catéter}

Sin ninguna duda, el aspecto más importante relacionado con las BRC en los próximos años será la prevención. Se puede acometer desde tres ángulos diferentes: dispositivos en las conexiones, composición de los catéteres y la aplicación de medidas estrictas y reconocidas tanto durante la inserción como en el mantenimiento de los catéteres. La dificultad de evaluar la eficacia de dispositivos estriba en que la reducción de la bacteriemia depende en gran medida de cuál es el punto de partida. Si se parte de tasas de bacteriemias muy elevadas, será relativamente fácil obtener beneficios, pero si se parte de tasas bajas, pequeñas reducciones pueden ser mucho más difíciles de conseguir. Además, casi nunca se aplica una medida sola, por lo que no es posible medir el beneficio de una medida aislada. Por ejemplo, con los dispositivos colocados en las conexiones, Esteve et $\mathrm{al}^{64}$ estudian la incidencia de BRC en pacientes ingresados en UCI en los que se emplean catéteres con un sistema de conexión valvular sin aguja y la comparan con la de las conexiones con llaves de tres pasos. No hay diferen- cias significativas en la tasas de BRC, ya que hubo 4,61 bacteriemias cada 1.000 días de uso de catéter en pacientes portadores de sistemas de conexión valvular, frente a 4,11/1.000 días de uso de catéter en pacientes en los que no se emplearon, por lo que se concluye que la utilización de estos dispositivos no reduce la incidencia de BRC.

En los últimos años se han publicado al menos una decena de metaanálisis y revisiones sistemáticas del papel de los catéteres impregnados de antisépticos o antibióticos en la prevención de la BTC. En la última de estas publicaciones Ramritu et $\mathrm{al}^{65}$ concluyen que los catéteres impregnados de clorhexidina o sulfadiazina de plata reducen el riesgo de $\mathrm{BRC}$ con respecto a los catéteres no recubiertos ( $\mathrm{RR}=0,66$; IC del 95\%, 0,47-0,93). Los catéteres impregnados de minociclina y rifampicina son más efectivos que los impregnados de antisépticos $(\mathrm{RR}=0,12$; IC del 95\%, 0,02$0,67)$ en la prevención de BTC. Sin embargo, la nueva generación (recubiertos en las superficies externa e interna) de catéteres impregnados en clorhexidina/ sulfadiazina de plata, en platino o en carbón (o mezcla de ellos) muestran una reducción no significativa del riesgo de $\mathrm{BRC}$ respecto a los no recubiertos. Los autores sostienen que se requieren otros estudios para el uso habitual de estos catéteres.

Tres ensayos clínicos recientes ${ }^{66-68}$ con diversos recubrimientos no han encontrado reducciones de la tasa de bacteriemia relacionada con catéteres, aunque sí en las de colonizaciones. Por el contrario, un estudio en el que se compararon las tasas de BRC antes y después de la introducción de catéteres impregnados con clorhexidina y sulfadiazina (estudiando dos periodos de 2 años cada uno dentro de una misma unidad) mostró una reducción del $35 \%$ en el número de BRC y un considerable ahorro económico ${ }^{69}$. El debate sobre la aplicación sistemática de estos catéteres en pacientes críticos continúa, y varios autores se han ocupado de este problema en diferentes metaanálisis y revisiones sistemáticas ${ }^{69-76}$. La mayoría de las opiniones de los expertos coincide en señalar la necesidad de implantar programas educativos sobre las recomendaciones clásicas, y solamente si no se puede disminuir la tasa de BRC considerar, en determinados pacientes, el empleo de catéteres impregnados; pero no se está de acuerdo al cuantificar la tasa mínima que asegurara que estos dispositivos sean coste-efectivos.

Un claro ejemplo de la eficacia de un programa educativo y la adopción de medidas sencillas fue publicado por Pronovost et $\mathrm{al}^{77}$. El seguimiento estricto del lavado de manos, la utilización de medidas de barrera, la desinfección de la piel con clorhexidina, evitar accesos femorales y retirar los catéteres en 103 unidades de cuidados intensivos del estado de Michigan redujo drásticamente la tasa de $\mathrm{BRC}$, y el efecto perduró (mediana de reducción, de 2,7 a 0,34 bacteriemias/1.000 días catéter). La próxima implantación de este programa en nuestro país esperamos que confirme esta tendencia.

Por último, es llamativo el resultado encontrado por Silvestri et al ${ }^{78}$ que en una revisión sistemática de 
estudios aleatorizados de descontaminación digestiva selectiva (más de 4.000 pacientes) encuentran una disminución del número de bacteriemias $(\mathrm{OR}=0,73$; IC del 95\%, 0,59-0,90; $\mathrm{p}=0,0036$; reducción absoluta, $37 \%$ ), pero exclusivamente las causadas por bacilos gramnegativos. Asimismo se encuentra una disminución de la mortalidad $(\mathrm{OR}=0,74$; $\mathrm{IC}$ del $95 \%, 0,61-0,91 ; \mathrm{p}=0,0034$; reducción absoluta, $4,6 \%)$. Cuando se analizaron exclusivamente las $\mathrm{BRC}$, hubo una reducción no significativa de las causadas por bacilos gramnegativos y ningún efecto en las causadas por grampositivos. Estas, en general, las causan patógenos de la piel, a la que no afecta la técnica de descontaminación digestiva. Los autores concluyen que sería necesario tratar a 20 pacientes para disminuir una bacteriemia por gramnegativos y a 22 para evitar una muerte.

En la tabla 3 se reflejan algunos mensajes extraídos de la literatura reciente sobre las bacteriemias.

\section{NUEVOS TRATAMIENTOS ANTIMICROBIANOS}

En la revisión de la literatura médica, llama la atención que en los últimos años se haya dedicado mucho más esfuerzo de investigación dirigida al tratamiento de infecciones por grampositivos que al tratamiento de bacterias gramnegativas multirresistentes como $P$. aerugionosa o Acinetobacter baumanii. Es bien patente la influencia del mercado americano, donde SARM y Enterococcus resistente a vancomicina constituyen un grave problema. Evidentemente, los artículos que en este apartado se mencionan no son los únicos publicados en el ámbito del tratamiento antibiótico en estos 2 años. Se han estudiado diferentes aspectos de toxicidad y eficacia de vancomicina, nuevas aplicaciones de colistina, combinaciones de antibióticos (rifampicina con colistina, por ejemplo), diferentes formas de administrar antibióticos (perfusión continua de betalactámicos o glucopéptidos, vía inhalatoria, etc.) o estrategias antibióticas dirigidas a reducir la aparición de multirresistencias; pero estos aspecto, aun siendo todos ellos interesantes, no van a ser objeto de la revisión de este apartado.

Hace aproximadamente 2 años se aprobó en nuestro país el empleo de tigeciclina. No se va hacer aquí una descripción de sus propiedades como antimicrobiano, sino constatar que empieza a aparecer literatura médica sobre su utilización en pacientes críticos. Swoboda et al $^{79}$ describen el empleo de tigeciclina en 70 pacientes con sepsis severa o shock séptico. Muchos de sus pacientes tienen comorbilidades como neoplasias o insuficiencia renal y se emplea este antibiótico sobre todo en el tratamiento de infecciones abdominales. En su serie predominan patógenos como SARM o enterococos resistentes a vancomicina. La mortalidad total fue del $30 \%$, lo que llama la atención a los autores por ser una cifra baja. La impresión general es que en España el empleo de tigeciclina se hace de distinta manera, buscando más el tratamiento de infecciones por Acinetobacter y quizá

\section{TABLA 3. Mensajes obtenidos de la revisión sistemática. Bacteriemia relacionada con catéter vascular (BRC)}

Epidemiología: Téngase en cuenta los bacilos gramnegativos y hongos en las vías femorales con sospecha de infección como causante de BRC

Diagnóstico de la infección por catéter: En la práctica clínica habitual en UCI, hay que seguir considerando los métodos clásicos

Prevención de BRC: Hay que adoptar las medidas reconocidas y recomendadas: lavado de manos, precauciones máximas de barrera, desinfección de la piel con clorhexidina, evitar la canalización de femorales y eliminar los catéteres innecesarios. Después hay que pensar en otros dispositivos (catéteres impregnados de antisépticos o antibióticos) si no se consigue reducir la tasa de BRC

más en neumonía, datos que hasta la fecha no han sido publicados en forma de artículo.

Más recientemente se ha aprobado el empleo de daptomicina, por lo que todavía no hay suficientes datos de su utilización en pacientes críticos. El trabajo de más importancia en lo que se refiere a nuestros pacientes es el de Fowler et $a^{80}$, que sirvió para su aprobación en el tratamiento de bacteriemias y endocarditis derecha. No se hace mención en dicho artículo a cuántos pacientes estaban ingresados en UCI en el momento del diagnóstico de bacteriemia o endocarditis, pero sus resultados, en principio, debían de ser aplicables a nuestros pacientes.

Ertapenem, otro de los antibióticos comercializados hace pocos años, podría tener un papel importante en pacientes críticos en el tratamiento de infecciones por bacilos gramnegativos productores de betalactamasa de espectro extendido, tal y como se ha demostrado en una corta serie de casos con neumonías causadas por estos microorganismos ${ }^{81}$.

Entre los antibióticos aún no comercializados, ceftobiprol es una cefalosporina con la particularidad de tener una amplia cobertura contra bacilos gramnegativos y cocos grampositivos -incluido SARM-, por lo que se ha probado en infecciones de partes blandas ${ }^{82}$ (estudio de no inferioridad frente a vancomicina más ceftazidima), con resultados similares a los obtenidos con los antibióticos con los que se lo comparó y sin mayor tasa de efectos secundarios. Aunque aún queda lejos su empleo en pacientes críticos, probablemente las siguientes investigaciones de este antibiótico se centrarán en el campo de la NAC y la neumonía hospitalaria.

Tres glucopéptidos están actualmente en estudio y tienen como peculiaridad una farmacocinética muy favorable y probablemente menos efectos secundarios que los actuales glucopéptidos. Entre telavanci$\mathrm{na}^{83}$, oritanvancina ${ }^{84}$ y dalbavancina ${ }^{85}$, destaca éste por la posibilidad de administrarlo en una sola dosis semanal, lo que hace muy tentador emplearlo en BRC. Entre las quinolonas, garenoxacino parece tener una potencia superior a la de levofloxacino y probablemente será el que lo sustituya en el futuro ${ }^{86}$, mientras que moxifloxacino en monoterapia, que se emplea actualmente por vía oral, se está estudiando 
también por vía intravenosa en la NAC, con resultados similares a la combinación de ceftriaxona y levofloxacino ${ }^{87}$.

Por último, recientemente se ha publicado el primer ensayo clínico de doripenem ${ }^{88}$, un carbapénemico con características similares a las del meropenem pero probablemente más activo contra $P$. aeruginosa. En un ensayo clínico ${ }^{88}$ se lo comparó con imipenem en NAVM, con resultados similares. Es de esperar, por lo tanto, que este antibiótico se apruebe próximamente para esta indicación.

\section{Conflicto de intereses}

El autor declara haber tenido colaboraciones en forma de conferencias financiadas por Novartis, Wyeth, MSD y Pfizer, pero ello no ha influido en la selección ni la exposición de la presente revisión.

\section{BIBLIOGRAFÍA}

1. Mandell LA, Wunderink RG, Anzueto A, Bartlett JG, Campbell GD, Dean NC, et al. Infectious Diseases Society of America/American Thoracic Society consensus guidelines on the management of community-acquired pneumonia in adults. Clin Infect Dis. 2007;44 Suppl 2:S27-72.

2. Bodi M, Rodriguez A, Sole-Violan J, Gilavert MC, Garnacho $\mathrm{J}$, Blanquer J, et al. Antibiotic prescription for community-acquired pneumonia in the intensive care unit: impact of adherence to Infectious Diseases Society of America guidelines on survival. Clin Infect Dis. 2005;41:1709-16.

3. Shorr AF, Bodi M, Rodriguez A, Sole-Violan J, GarnachoMontero J, Rello J. Impact of antibiotic guideline compliance on duration of mechanical ventilation in critically ill patients with community-acquired pneumonia. Chest. 2006;130:93-100.

4. Mortensen EM, Restrepo MI, Anzueto A, Pugh JA. Antibiotic therapy and 48-hour mortality for patients with pneumonia. Am J Med. 2006;119:859-64.

5. Calzada SR, Tomas RM, Romero MJ, Moragon EM, Cataluna JJ, Villanueva RM. Empiric treatment in hospitalized communityacquired pneumonia. Impact on mortality, length of stay and readmission. Respir Med. 2007;101:1909-15.

6. Dean NC, Bateman KA, Donnelly SM, Silver MP, Snow GL, Hale D. Improved clinical outcomes with utilization of a community-acquired pneumonia guideline. Chest. 2006;130:794-9.

7. Valencia M, Badia JR, Cavalcanti M, Ferrer M, Agusti C, Angrill J, et al. Pneumonia severity index class v patients with community-acquired pneumonia: characteristics, outcomes, and value of severity scores. Chest. 2007;132:515-22.

8. Marrie TJ, Shariatzadeh MR. Community-acquired pneumonia requiring admission to an intensive care unit: a descriptive study. Medicine (Baltimore). 2007;86:103-11.

9. Restrepo MI, Mortensen EM, Velez JA, Frei C, Anzueto A. A comparative study of community-acquired pneumonia patients admitted to the ward and the ICU. Chest. 2008;133:610-7.

10. Shorr AF, Wunderink RG. There is no «cap» on the importance of community-acquired pneumonia in the ICU. Chest. 2008;133:590-2.

11. Guidelines for the management of adults with hospital-acquired, ventilator-associated, and healthcare-associated pneumonia. Am J Respir Crit Care Med. 2005;171:388-416.

12. Micek ST, Heuring TJ, Hollands JM, Shah RA, Kollef MH. Optimizing antibiotic treatment for ventilator-associated pneumonia. Pharmacotherapy. 2006;26:204-13.

13. Carratala J, Mykietiuk A, Fernandez-Sabe N, Suarez C, Dorca J, Verdaguer R, et al. Health care-associated pneumonia requiring hospital admission: epidemiology, antibiotic therapy, and clinical outcomes. Arch Intern Med. 2007;167:1393-9.
14. Alfageme I, Aspa J, Bello S, Blanquer J, Blanquer R, Borderias L, et al. Guías para el diagnóstico y tratamiento de la neumonía adquirida en la comunidad. Sociedad Española de Neumología y Cirugía Torácica (SEPAR). Arch Bronconeumol. 2005:41:272-89.

15. Rodriguez A, Mendia A, Sirvent JM, Barcenilla F, De la Torre-Prados MV, Sole-Violan J, et al. Combination antibiotic therapy improves survival in patients with community-acquired pneumonia and shock. Crit Care Med. 2007;35:1493-8.

16. Robenshtok E, Shefet D, Gafter-Gvili A, Paul M, Vidal L, Leibovici L. Empiric antibiotic coverage of atypical pathogens for community acquired pneumonia in hospitalized adults. Cochrane Database Syst Rev. 2008;CD004418.

17. Mortensen EM, Restrepo MI, Anzueto A, Pugh J. The impact of empiric antimicrobial therapy with a beta-lactam and fluoroquinolone on mortality for patients hospitalized with severe pneumonia. Crit Care. 2005;10:R8

18. Lodise TP, Kwa A, Cosler L, Gupta R, Smith RP. Comparison of beta-lactam and macrolide combination therapy versus fluoroquinolone monotherapy in hospitalized Veterans Affairs patients with community-acquired pneumonia. Antimicrob Agents Chemother. 2007;51:3977-82.

19. Metersky ML, Ma A, Houck PM, Bratzler DW. Antibiotics for bacteremic pneumonia: Improved outcomes with macrolides but not fluoroquinolones. Chest. 2007;131:466-73.

20. Aspa J, Rajas O, Rodriguez C, Huertas MC, Borderias L, Cabello FJ, et al. Impact of initial antibiotic choice on mortality from pneumococcal pneumonia. Eur Respir J. 2006;27:1010-9.

21. Giamarellos-Bourboulis EJ, Pechere JC, Routsi C, Plachouras D, Kollias S, Raftogiannis M, et al. Effect of clarithromycin in patients with sepsis and ventilator-associated pneumonia. Clin Infect Dis. 2008;46:1157-64.

22. The Canadian Critical Care Trials Group. A randomized trial of diagnostic techniques for ventilator-associated pneumonia. N Engl J Med. 2006;355:2619-30.

23. Giantsou E, Liratzopoulos N, Efraimidou E, Panopoulou M, Alepopoulou E, Kartali-Ktenidou S, et al. De-escalation therapy rates are significantly higher by bronchoalveolar lavage than by tracheal aspirate. Intensive Care Med. 2007:33:1533-40.

24. Kuti EL, Patel AA, Coleman CI. Impact of inappropriate antibiotic therapy on mortality in patients with ventilator-associated pneumonia and blood stream infection: a meta-analysis. J Crit Care. 2008;23:91-100.

25. Gastmeier P, Sohr D, Geffers C, Behnke M, Ruden H. Risk factors for death due to nosocomial infection in intensive care unit patients: findings from the Krankenhaus Infektions Surveillance System. Infect Control Hosp Epidemiol. 2007;28:466-72.

26. Medina J, Formento C, Pontet J, Curbelo A, Bazet C, Gerez $\mathrm{J}$, et al. Prospective study of risk factors for ventilator-associated pneumonia caused by Acinetobacter species. J Crit Care. 2007;22:18-26.

27. Aloush V, Navon-Venezia S, Seigman-Igra Y, Cabili S, Carmeli Y. Multidrug-resistant Pseudomonas aeruginosa: risk factors and clinical impact. Antimicrob Agents Chemother. 2006;50:43-8

28. Parker CM, Kutsogiannis J, Muscedere J, Cook D, Dodek P, Day AG, et al. Ventilator-associated pneumonia caused by multidrug-resistant organisms or Pseudomonas aeruginosa: prevalence, incidence, risk factors, and outcomes. J Crit Care. 2008;23:18-26.

29. Rello J, Allegri C, Rodriguez A, Vidaur L, Sirgo G, Gomez F, et al. Risk factors for ventilator-associated pneumonia by Pseudomonas aeruginosa in presence of recent antibiotic exposure. Anesthesiology. 2006;105:709-14

30. Georges B, Conil JM, Dubouix A, Archambaud M, Bonnet E, Saivin S, et al. Risk of emergence of Pseudomonas aeruginosa resistance to beta-lactam antibiotics in intensive care units. Crit Care Med. 2006;34:1636-41.

31. Nseir S, Di Pompeo C, Jozefowicz E, Cavestri B, Brisson H, Nyunga M, et al. Relationship between tracheotomy and ventilator-associated pneumonia: a case control study. Eur Respir J. 2007;30:314-20.

32. Gasink LB, Fishman NO, Nachamkin I, Bilker WB, Lautenbach E. Risk factors for and impact of infection or colonization with aztreonam-resistant Pseudomonas aeruginosa. Infect Control Hosp Epidemiol. 2007;28:1175-80. 
33. Fujimura S, Nakano Y, Sato T, Shirahata K, Watanabe A. Relationship between the usage of carbapenem antibiotics and the incidence of imipenem-resistant Pseudomonas aeruginosa. J Infect Chemother. 2007; 13:147-50.

34. Lodise TP, Miller CD, Graves J, Furuno JP, McGregor JC Lomaestro B, et al. Clinical prediction tool to identify patients with Pseudomonas aeruginosa respiratory tract infections at greatest risk for multidrug resistance. Antimicrob Agents Chemother. 2007;51:417-22.

35. Oztoprak N, Cevik MA, Akinci E, Korkmaz M, Erbay A Eren SS, et al. Risk factors for ICU-acquired methicillin-resistant Staphylococcus aureus infections. Am J Infect Control. 2006;34:1-5.

36. Álvarez-Lerma F, Palomar M, Olaechea P, Otal JJ, Insausti J, Cerdá E. Estudio Nacional de Vigilancia de Infección Nosocomial en Unidades de Cuidados Intensivos. Informe evolutivo de los años 2003-2005. Med Intensiva. 2007;31:6-17

37. Aarts MA, Hancock JN, Heyland D, McLeod RS, Marshall JC. Empiric antibiotic therapy for suspected ventilator-associated pneumonia: a systematic review and meta-analysis of randomized trials. Crit Care Med. 2008;36:108-17.

38. Garnacho-Montero J, Sa-Borges M, Sole-Violan J, Barcenilla F, Escoresca-Ortega A, Ochoa M, et al. Optimal management therapy for Pseudomonas aeruginosa ventilator-associated pneumonia: an observational, multicenter study comparing monotherapy with combination antibiotic therapy. Crit Care Med. 2007;35:188895

39. Heyland DK, Dodek P, Muscedere J, Day A, Cook D. Randomized trial of combination versus monotherapy for the empiric treatment of suspected ventilator-associated pneumonia. Crit Care Med. 2008;36:737-44.

40. Muscedere J, Dodek P, Keenan S, Fowler R, Cook D, Heyland D. Comprehensive evidence-based clinical practice guidelines for ventilator-associated pneumonia: diagnosis and treatment. J Crit Care. 2008;23:138-47.

41. Pineda LA, Saliba RG, El Solh AA. Effect of oral decontamination with chlorhexidine on the incidence of nosocomial pneumonia: a meta-analysis. Crit Care. 2006;10:R35.

42. Chlebicki MP, Safdar N. Topical chlorhexidine for prevention of ventilator-associated pneumonia: a meta-analysis. Crit Care Med. 2007;35:595-602.

43. Chan EY, Ruest A, Meade MO, Cook DJ. Oral decontamination for prevention of pneumonia in mechanically ventilated adults: systematic review and meta-analysis. BMJ. 2007:334:889.

44. Segers $P$, Speekenbrink RG, Ubbink DT, Van Ogtrop ML, De Mol BA. Prevention of nosocomial infection in cardiac surgery by decontamination of the nasopharynx and oropharynx with chlorhexidine gluconate: a randomized controlled trial. JAMA. 2006;296:2460-6

45. Koeman M, Van der Ven AJ, Hak E, Joore HC, Kaasjager K, De Smet AG, et al. Oral decontamination with chlorhexidine reduces the incidence of ventilator-associated pneumonia. Am J RespirCrit Care Med. 2006;173:1348-55

46. Mori H, Hirasawa H, Oda S, Shiga H, Matsuda K, Nakamura M. Oral care reduces incidence of ventilator-associated pneumonia in ICU populations. Intensive Care Med. 2006;32:230-6.

47. Taylor N, Van Saene HK, Abella A, Silvestri L, Vucic M, Peric M. Descontaminación digestiva selectiva. ¿Por qué no aplicamos la evidencia en la práctica clínica? Med Intensiva. 2007:31:136-45.

48. Stoutenbeek CP, Van Saene HK, Little RA, Whitehead A. The effect of selective decontamination of the digestive tract on mortality in multiple trauma patients: a multicenter randomized controlled trial. Intensive Care Med. 2007;33:261-70.

49. Muscedere J, Dodek P, Keenan S, Fowler R, Cook D, Heyland D. Comprehensive evidence-based clinical practice guidelines for ventilator-associated pneumonia: prevention. J Crit Care. 2008;23:126-37.

50. Heininger A, Meyer E, Schwab F, Marschal M, Unertl K, Krueger WA. Effects of long-term routine use of selective digestive decontamination on antimicrobial resistance. Intensive Care Med. 2006;32:1569-76

51. Al Naiemi N, Heddema ER, Bart A, De Jonge E, Vandenbroucke-Grauls CM, Savelkoul PH, et al. Emergence of multidrug-resistant Gram-negative bacteria during selective de- contamination of the digestive tract on an intensive care unit. J Antimicrob Chemother. 2006;58:853-6.

52. Niel-Weise BS, Snoeren RL, Van den Broek PJ. Policies for endotracheal suctioning of patients receiving mechanical ventilation: a systematic review of randomized controlled trials. Infect Control Hosp Epidemiol. 2007;28:531-6.

53. Siempos II, Vardakas KZ, Falagas ME. Closed tracheal suction systems for prevention of ventilator-associated pneumonia. $\mathrm{Br}$ J Anaesth. 2008;100:299-306.

54. Vonberg RP, Eckmanns $T$, Welte $T$, Gastmeier P. Impact of the suctioning system (open vs. closed) on the incidence of ventilation-associated pneumonia: Meta-analysis of randomized controlled trials. Intensive Care Med. 2006;32:1329-35.

55. Jongerden IP, Rovers MM, Grypdonck MH, Bonten MJ. Open and closed endotracheal suction systems in mechanically ventilated intensive care patients: a meta-analysis. Crit Care Med. 2007;35:260-70

56. Lorente L, Lecuona M, Jimenez A, Mora ML, Sierra A. Influence of an endotracheal tube with polyurethane cuff and subglottic secretion drainage on pneumonia. Am J Respir Crit Care Med. 2007;176:1079-83.

57. Poelaert J, Depuydt P, De Wolf A, Van d V, Herck I, Blot S. Polyurethane cuffed endotracheal tubes to prevent early postoperative pneumonia after cardiac surgery: a pilot study. J Thorac Cardiovasc Surg. 2008;135:771-6.

58. Rello J, Kollef M, Diaz E, Sandiumenge A, Del Castillo Y, Corbella X, et al. Reduced burden of bacterial airway colonization with a novel silver-coated endotracheal tube in a randomized multiple-center feasibility study. Crit Care Med. 2006;34:2766-72.

59. Gastmeier P, Geffers C. Prevention of ventilator-associated pneumonia: analysis of studies published since 2004. J Hosp Infect. 2007;67:1-8.

60. Lorente L, Blot S, Rello J. Evidence on measures for the prevention of ventilator-associated pneumonia. Eur Respir J. 2007:30:1193-207

61. Lorente L, Jimenez A, Santana M, Iribarren JL, Jimenez JJ, Martin MM, et al. Microorganisms responsible for intravascular catheter-related bloodstream infection according to the catheter site. Crit Care Med. 2007:35:2424-7.

62. Bouza E, Alvarado N, Alcala L, Munoz P, Rabadan PM, Rodriguez-Creixems M. An instant procedure to demonstrate catheter-tip colonization may help clinicians. Diagn Microbiol Infect Dis. 2006;56:255-60

63. Bouza E, Alvarado N, Alcala L, Perez MJ, Rincon C, Munoz $\mathrm{P}$. A randomized and prospective study of 3 procedures for the diagnosis of catheter-related bloodstream infection without catheter withdrawal. Clin Infect Dis. 2007;44:820-6.

64. Esteve F, Pujol M, Limon E, Saballs M, Argerich MJ, Verdaguer R, et al. Bloodstream infection related to catheter connections: a prospective trial of two connection systems. J Hosp Infect. 2007;67:30-4

65. Ramritu P, Halton K, Cook D, Whitby M, Graves N. Catheter-related bloodstream infections in intensive care units: a systematic review with meta-analysis. J Adv Nurs. 2008;62:3-21.

66. Kalfon P, De Vaumas C, Samba D, Boulet E, Lefrant JY, Eyraud D, et al. Comparison of silver-impregnated with standard multi-lumen central venous catheters in critically ill patients. Crit Care Med. 2007;35:1032-9.

67. Osma S, Kahveci SF, Kaya FN, Akalin H, Ozakin C, Yilmaz E, et al. Efficacy of antiseptic-impregnated catheters on catheter colonization and catheter-related bloodstream infectio$\mathrm{ns}$ in patients in an intensive care unit. J Hosp Infect. 2006; 62:156-62.

68. Fraenkel D, Rickard C, Thomas P, Faoagali J, George N, Ware R. A prospective, randomized trial of rifampicin-minocycline-coated and silver-platinum-carbon-impregnated central venous catheters. Crit Care Med. 2006;34:668-75.

69. Borschel DM, Chenoweth CE, Kaufman SR, Hyde KV, VanDerElzen KA, Raghunathan TE, et al. Are antiseptic-coated central venous catheters effective in a real-world setting? Am J Infect Control. 2006;34:388-93.

70. Gastmeier P, Geffers C. Prevention of catheter-related bloodstream infections: analysis of studies published between 2002 and 2005. J Hosp Infect. 2006;64:326-35 
71. Niel-Weise BS, Stijnen T, Van den Broek PJ. Anti-infectivetreated central venous catheters: a systematic review of randomized controlled trials. Intensive Care Med. 2007;33:2058-68.

72. Niel-Weise BS, Stijnen T, Van den Broek PJ. Anti-infectivetreated central venous catheters for total parenteral nutrition or chemotherapy: a systematic review. J Hosp Infect. 2008;69:11423.

73. Gilbert RE, Harden M. Effectiveness of impregnated central venous catheters for catheter related blood stream infection: a systematic review. Curr Opin Infect Dis. 2008;21:235-45.

74. Raad I, Hanna H, Maki D. Intravascular catheter-related infections: advances in diagnosis, prevention, and management. Lancet Infect Dis. 2007;7:645-57.

75. Eggimann P. Prevention of intravascular catheter infection. Curr Opin Infect Dis. 2007;20:360-9.

76. Timsit JF. Diagnosis and prevention of catheter-related infections. Curr Opin Crit Care. 2007;13:563-71.

77. Pronovost P, Needham D, Berenholtz S, Sinopoli D, Chu H, Cosgrove $\mathrm{S}$, et al. An intervention to decrease catheter-related bloodstream infections in the ICU. N Engl J Med. 2006;355:272532 .

78. Silvestri L, Van Saene HK, Milanese M, Gregori D, Gullo A. Selective decontamination of the digestive tract reduces bacterial bloodstream infection and mortality in critically ill patients Systematic review of randomized, controlled trials. J Hosp Infect. 2007:65:187-203.

79. Swoboda S, Ober M, Hainer C, Lichtenstern C, Seiler C, Wendt C, et al. Tigecycline for the treatment of patients with severe sepsis or septic shock: a drug use evaluation in a surgical intensive care unit. J Antimicrob Chemother. 2008;61:729-33.

80. Fowler VG Jr, Boucher HW, Corey GR, Abrutyn E, Karchmer AW, Rupp ME, et al. Daptomycin versus standard therapy for bacteremia and endocarditis caused by Staphylococcus aureus. N Engl J Med. 2006;355:653-65.
81. Bassetti M, Righi E, Fasce R, Molinari MP, Rosso R, Di Biagio A, et al. Efficacy of ertapenem in the treatment of early ventilator-associated pneumonia caused by extended-spectrum beta-lactamase-producing organisms in an intensive care unit. $\mathrm{J}$ Antimicrob Chemother. 2007:60:433-5.

82. Noel GJ, Bush K, Bagchi P, Ianus J, Strauss RS. A randomized, double-blind trial comparing ceftobiprole medocaril with vancomycin plus ceftazidime for the treatment of patients with complicated skin and skin-structure infections. Clin Infect Dis. 2008;46:647-55.

83. Stryjewski ME, Chu VH, O'Riordan WD, Warren BL, Dunbar LM, Young DM, et al. Telavancin versus standard therapy for treatment of complicated skin and skin structure infections caused by gram-positive bacteria: FAST 2 study. Antimicrob Agents Chemother. 2006;50:862-7.

84. Bhavnani SM, Passarell JA, Owen JS, Loutit JS, Porter SB, Ambrose PG. Pharmacokinetic-pharmacodynamic relationships describing the efficacy of oritavancin in patients with Staphylococcus aureus bacteremia. Antimicrob Agents Chemother. 2006;50:994-1000.

85. Chen AY, Zervos MJ, Vazquez JA. Dalbavancin: a novel antimicrobial. Int J Clin Pract. 2007;61:853-63.

86. Jones RN, Sader HS, Stilwell MG, Fritsche TR. Garenoxacin activity against isolates form patients hospitalized with community-acquired pneumonia and multidrug-resistant Streptococcus pneumoniae. Diagn Microbiol Infect Dis. 2007:58:1-7.

87. Torres A, Garau J, Arvis P, Carlet J, Choudhri S, Kureishi A, et al. Moxifloxacin monotherapy is effective in hospitalized patients with community-acquired pneumonia: the MOTIV study -a randomized clinical trial. Clin Infect Dis. 2008;46:1499-509.

88. Chastre J, Wunderink R, Prokocimer P, Lee M, Kaniga K, Friedland I. Efficacy and safety of intravenous infusion of doripenem versus imipenem in ventilator-associated pneumonia: a multicenter, randomized study. Crit Care Med. 2008;36:1089-96. 